\title{
AREA THEOREMS AND FREDHOLM EIGENVALUES
}

\section{Erich Hoy}

\section{Introduction}

In this paper we shall derive an area theorem for conformal mappings onto a domain whose Fredholm eigenvalue is bounded from below. Furthermore, we will prove the following extremum property of circular rings: The smallest nontrivial Fredholm eigenvalue of a doubly connected domain having a fixed conformal modulus attains its maximal value if, and only if, the boundary of the domain consists of two circles.

Let $G$ be an unbounded plane domain bounded by $n$ closed analytic Jordan curves such that the complement $G^{C}$ of $G$ consists of simply connected closed regions. We denote by $\sum(G)$ the class of analytic and univalent functions $f(z)$ $(z \in G \backslash\{\infty\})$ having the Laurent series expansion

$$
f(z)=z+\alpha_{0}+\frac{\alpha_{1}}{z}+\frac{\alpha_{2}}{z^{2}}+\cdots
$$

at infinity. Let $\sum\langle G, \kappa\rangle$ be the subclass of $\sum(G)$ containing all functions $f(z)$ for which the domain $G^{*}=f(G)$ has the smallest non-trivial Fredholm eigenvalue $\lambda_{2}$ satisfying

$$
\lambda_{2} \geq \frac{1}{\kappa}
$$

where the positive number $\kappa<1$ is fixed.

A classical definition of the Fredholm eigenvalue $\lambda_{2}$ is contained in [4; p. $3 \mathrm{ff}$.]. For our purposes we need the generalized definition of $\lambda_{2}$ given by G. Springer. We denote by $G^{*}$ an unbounded domain of finite connectivity bounded by closed pairwise disjoint Jordan curves. Then the Fredholm eigenvalue $\lambda_{2}$ of $G^{*}$ is the greatest number $\lambda>1$ satisfying

$$
\frac{\lambda+1}{\lambda-1} \geq \frac{\iint_{G^{*}}[\nabla H(w)]^{2} d u d v}{\iint_{G^{*}}[\nabla H(w)]^{2} d u d v} \geq \frac{\lambda-1}{\lambda+1}, \quad(w=u+i v)
$$

for all continuous functions $H(w)$ which are harmonic in $G^{*}$ and in the interior of $G^{* C}$ and have a single-valued harmonic conjugate (see [21] and cf. [1], [3], [10] 
and [19]). Note that $H(w)$ may be a real or a complex harmonic function. In the latter case the expression $[\nabla H(w)]^{2}$ means $\left|H_{u}(w)\right|^{2}+\left|H_{v}(w)\right|^{2}$.

Let $\left\{\varphi_{\nu}(z), \Phi_{\nu}(z)\right\},(\nu=1,2, \ldots)$ be the orthonormal system of functions fulfilling

$$
\overline{d \varphi_{\nu}(z)}=d \Phi_{\nu}(z)
$$

on the boundary $\partial G$ of $G$. The functions $\varphi_{\nu}(z)$ are analytic in the closure of $G$. The functions $\Phi_{\nu}(z)$ have a pole at infinity and are analytic for all finite $z$ in the closure of $G$. This system of functions is introduced in [16]. The functions of this orthonormal system are closely related to mappings onto parallel slit domains. For example, $\Phi_{1}(z)+\varphi_{1}(z)$ and $\Phi_{1}(z)-\varphi_{1}(z)$ map $G$ univalently onto parallel slit domains.

Besides (4), we need another main property of this system. If $T(w)$ is an analytic function in the complement of $f(G)$ for all mappings $f(z) \in \sum(G)$, then

$$
\{T[f(z)]\}^{\prime}=\sum_{\nu=1}^{\infty} \Gamma_{\nu} \Phi_{\nu}^{\prime}(z)+\sum_{\nu=1}^{\infty} \gamma_{\nu} \varphi_{\nu}^{\prime}(z)
$$

holds for all $z \in G$ in a sufficiently small neighbourhood of points on $\partial G$ and for all mappings $f(z) \in \sum(G)$. A simple example of such a function $T(w)$ is $T(w)=w$. In this case we have

$$
f^{\prime}(z)=a_{11} \Phi_{1}^{\prime}(z)+\sum_{\nu=1}^{\infty} \gamma_{\nu} \varphi_{\nu}^{\prime}(z)
$$

where $a_{11}>0$ is a fixed number depending only on the domain $G$. Besides, $a_{11}^{2}$ represents the radius of that circular disk which is the exact range of the coefficient $\alpha_{1}$ in (1) when $f(z)$ belongs to the class $\sum(G)$.

Finally, it should be noted that

$$
\iint_{[f(G)]^{C}}\left|T^{\prime}(w)\right|^{2} d u d v=\pi\left(\sum_{\nu=1}^{\infty}\left|\Gamma_{\nu}\right|^{2}-\sum_{\nu=1}^{\infty}\left|\gamma_{\nu}\right|^{2}\right) \quad(w=u+i v) .
$$

This follows from (5) for all mappings $f(z) \in \sum(G)$ (see [16; Theorem 4.13]).

\section{Derivation of an area theorem}

The basic idea of the following investigations is taken from [13]. We consider a continuous function $H(w)$ defined by

(7)

$$
\nabla H(w)= \begin{cases}\nabla \operatorname{Re}\left(e^{-i \theta} T(w)\right) & \text { if } w \in(f(G))^{C}, \\ \nabla \operatorname{Re}\left(e^{-i \theta}\left(T(w)-\sum \Gamma_{\nu} \Phi_{\nu}(z)\right)+e^{i \theta} \sum \bar{\Gamma}_{\nu} \varphi_{\nu}(z)\right) & \text { if } w=f(z) \in f(G),\end{cases}
$$


where $\theta$ is an arbitrary given number satisfying $0 \leq \theta<\pi$ and $\Gamma_{\nu}$ are the coefficients in (5). Since $f(z) \in \sum\langle G, \kappa\rangle$, we write (3) in the form

$$
\iint_{[f(G)]^{C}}(\nabla H(w))^{2} d u d v \geq \frac{1-\kappa}{1+\kappa} \iint_{f(G)}(\nabla H(w))^{2} d u d v
$$

The first integral in (8) is the same as

$$
\iint_{[f(G)]^{C}}\left|T^{\prime}(w)\right|^{2} d u d v
$$

By (6) the value of this integral is equal to

$$
\pi\left(\sum_{\nu=1}^{\infty}\left|\Gamma_{\nu}\right|^{2}-\sum_{\nu=1}^{\infty}\left|\gamma_{\nu}\right|^{2}\right)
$$

After a transformation into the $z$-plane we find for the other integral in (8) that

$$
\iint_{f(G)}(\nabla H(w))^{2} d u d v=\pi \sum_{\nu=1}^{\infty}\left|\gamma_{\nu}-e^{2 i \theta} \bar{\Gamma}_{\nu}\right|^{2} .
$$

Now we state our first result.

Theorem 1. Let $\theta$ and $\kappa$ be arbitrary numbers satisfying $0 \leq \theta<\pi$ and $0<\kappa<1$, respectively. If $f(z) \in \sum\langle G, \kappa\rangle$ then

$$
\sum_{\nu=1}^{\infty}\left|\gamma_{\nu}+\frac{1}{2}(1-\kappa) \bar{\Gamma}_{\nu} e^{2 i \theta}\right|^{2} \leq \frac{1}{4}(1+\kappa)^{2} \sum_{\nu=1}^{\infty}\left|\Gamma_{\nu}\right|^{2}
$$

holds for the coefficients $\Gamma_{\nu}, \gamma_{\nu}(\nu=1,2, \ldots)$ in (5).

In the next section we shall give some examples for Theorem 1.

\section{Examples}

Let $G$ be the exterior of the unit circle and $T(w)=w$. In this case we write $\sum\langle\kappa\rangle$ instead of $\sum\langle G, \kappa\rangle$ (see also [13] and [17; p. $\left.287 \mathrm{ff}.\right]$ ). Then (11) has the form

$$
\left(\left|\alpha_{1}\right|+\frac{1}{2}(1-\kappa)\right)^{2}+\sum_{\nu=2}^{\infty} \nu\left|\alpha_{\nu}\right|^{2} \leq \frac{1}{4}(1+\kappa)^{2} .
$$

The coefficients $\alpha_{\nu}(\nu=1,2, \ldots)$ are given in (1). The same inequality was proved by L.V. Ahlfors in [2] for the mappings of the known class $\sum(Q)$ where $\kappa$ and $Q$ satisfy

$$
\kappa=\frac{Q-1}{Q+1}
$$


But our class $\sum\langle\kappa\rangle$ is much wider than $\sum(Q)$ (see [13]). A better inequality for the class $\sum\langle\kappa\rangle$ than (12), namely

$$
\sum_{\nu=1}^{\infty} \nu\left|\alpha_{\nu}\right|^{2} \leq \kappa^{2}
$$

has been derived in [13]. (14) and also (12) imply that

$$
\left|\alpha_{1}\right| \leq \kappa
$$

Equality in (12), (14) and (15) holds for the mappings

$$
f^{*}(z)= \begin{cases}z+\kappa e^{2 i \alpha} / z+\text { const } & \text { if }|z|>1 \\ z+\kappa e^{2 i \alpha} \bar{z}+\text { const } & \text { if }|z| \leq 1\end{cases}
$$

where $\alpha$ is an arbitrary number satisfying $0 \leq \alpha<\pi$. It should be noted that $f^{*}(z) \in \sum\langle\kappa\rangle$ because the Fredholm eigenvalue $\lambda_{2}$ (in the classical sense) of an ellipse

$$
E=\left\{(x, y): \frac{x^{2}}{(1+\kappa)^{2}}+\frac{y^{2}}{(1-\kappa)^{2}}=1\right\}
$$

is exactly $1 / \kappa$ (see [19]).

Now let $G$ be a doubly connected domain. In this case it is more difficult to consider an explicit example because the system $\left\{\varphi_{\nu}(z), \Phi_{\nu}(z)\right\}(\nu=1,2, \ldots)$ is, in general, not available. Therefore, we shall investigate conformal mappings of an annulus $\{z: R<|z|<1 / R\}(0<R<1)$ onto an unbounded domain having the Fredholm eigenvalue $\lambda_{2} \geq 1 / \kappa$. For the sake of simplicity we propose $f(1)=\infty$ and

$$
f(z)-\frac{1}{z-1}=\sum_{\nu=-\infty}^{\infty} \delta_{\nu} z^{\nu} \quad(R<|z|<1 / R)
$$

Furthermore, we need a modification of $(7)$. Letting $T(w)=w$, it is necessary to find a harmonic function in the annulus with the boundary values $1 /(\bar{z}-1)$. Starting from the following expression with some unknown numbers $s_{\nu}^{(1)}$ and $s_{\nu}^{(2)}$ $(\nu \in \mathbf{Z})$

$$
\frac{1}{\bar{z}-1}=\sum_{\substack{\nu=-\infty \\ \nu \neq 0}}^{\infty}\left(s_{\nu}^{(1)} z^{\nu}+s_{\nu}^{(2)} \bar{z}^{\nu}\right)+s_{0}^{(1)} \ln |z|+s_{0}^{(2)}, \quad|z|=R, 1 / R,
$$

and making use of $z \cdot \bar{z}=R^{2}$ or $1 / R^{2}$ on the boundary of the annnulus we get 


$$
\begin{gathered}
s_{\nu}^{(1)}=-\frac{R^{2 \nu}}{R^{4 \nu}-1}, \\
s_{\nu}^{(2)}= \begin{cases}\left(R^{-4 \nu}-1\right)^{-1}, & \text { when } \nu>0, \\
\left(1-R^{4 \nu}\right)^{-1}, & \text { when } \nu<0,\end{cases}
\end{gathered}
$$

with $\nu \in \mathbf{Z} \backslash\{0\}$. Then we define

$$
\nabla H(w)=\nabla \operatorname{Re}\left(e^{-i \theta}\left(f(z)-\frac{1}{z-1}\right)+e^{i \theta} \sum_{\substack{\nu=-\infty \\ \nu \neq 0}}^{\infty}\left(s_{\nu}^{(1)} z^{\nu}+s_{\nu}^{(2)} \bar{z}^{\nu}\right)\right)
$$

when $w=f(z) \in f(G)$. Thus we obtain

$$
\begin{aligned}
\sum_{\nu=1}^{\infty} \nu \frac{1-R^{4 \nu}}{R^{2 \nu}} & \left(\left|\delta_{\nu}+\frac{R^{4 \nu}}{1-R^{4 \nu}}+\frac{1}{2}(1-\kappa) \frac{R^{2 \nu}}{1-R^{4 \nu}} e^{2 i \theta}\right|^{2}\right. \\
& \left.+\left|\delta_{-\nu}-\frac{R^{4 \nu}}{1-R^{4 \nu}}-\frac{1}{2}(1-\kappa) \frac{R^{2 \nu}}{1-R^{4 \nu}} e^{2 i \theta}\right|^{2}\right) \\
& \leq \frac{1}{2}(1+\kappa)^{2} \sum_{\nu=1}^{\infty} \nu \frac{R^{2 \nu}}{1-R^{4 \nu}},
\end{aligned}
$$

where the number $\theta$ may be arbitrarily chosen as before.

Multiply connected domains provide another difficulty. We will show in Section 4 that $\sum\langle G, \kappa\rangle$ is empty for too small numbers $\kappa>0$. To ensure that $\sum\langle G, \kappa\rangle \neq \emptyset$, we choose $\kappa \geq R^{2}$ because $1 / R^{2}$ is the Fredholm eigenvalue $\lambda_{2}$ of the given annulus $G$ (see [20]). This difficulty illustrates a remarkable property of a circle, namely $\lambda_{2}=\infty$. Hence, the identical mapping is always a member of $\sum\langle\kappa\rangle$ for all $\kappa \in(0,1)$, consequently $\sum\langle\kappa\rangle \neq \emptyset$.

In what follows we will investigate for which mappings $f(z) \in \sum\langle G, \kappa\rangle$ equality holds in (11).

We propose that for all mappings $f(z) \in \sum$ the function $T(f(z))-T(z)$ is analytic in $\{z:|z|>1\}$. In other words, the singularities of $T(f(z))$ do not depend on $f(z)$. Consequently, we have

$$
T(f(z))=\sum_{\nu=1}^{\infty} \Omega_{\nu} z^{\nu}+\text { const }+\sum_{\nu=1}^{\infty} \omega_{\nu} z^{-\nu}, \quad 1<|z|<r
$$

for $r$ sufficiently close to 1 . The numbers $\Omega_{\nu}, \nu=1,2, \ldots$, depend only on our choice of $T(w)$, and (18) is valid for all functions $f(z) \in \sum$. In particular, the area theorem for the class $\sum\langle\kappa\rangle$ may be written in the form

$$
\sum_{\nu=1}^{\infty} \nu\left|\omega_{\nu}+\frac{1}{2}(1-\kappa) e^{2 i \theta} \bar{\Omega}_{\nu}\right|^{2} \leq\left(\frac{1}{2}(1+\kappa)\right)^{2} \sum_{\nu=1}^{\infty} \nu\left|\Omega_{\nu}\right|^{2} .
$$


Firstly, we give an example for a mapping $f^{*}(z)$ for which equality holds in (19). In the following step it is shown that $f^{*}(z)$ belongs to $\sum\langle\kappa\rangle$. Defining a quasiconformal mapping $f^{*}(z)$ of the whole plane by

$$
f_{\bar{z}}^{*}(z)=\kappa \overline{\frac{T^{\prime}(z)}{T^{\prime}(z)}} f_{z}^{*}(z), \quad|z|<1
$$

and $f^{*}(z) \in \sum$, we conclude that $T\left(f^{*}(z)\right)$ also satisfies (20) for $|z|<1$. Consequently, the function

$$
F(z)= \begin{cases}T\left(f^{*}(z)\right)-T(z)-\kappa \overline{T(1 / \bar{z})}, & \text { if }|z|>1 \\ T\left(f^{*}(z)\right)-T(z)-\kappa \overline{T(z)}, & \text { if }|z| \leq 1\end{cases}
$$

is a solution of (20) which is analytic in the exterior of the unit circle. Hence, $F(z)$ must be a constant. Since $T(z)$ is an analytic function in the interior of the unit circle, (18) implies that

$$
T^{\prime}(z)=\sum_{\nu=1}^{\infty} \nu \Omega_{\nu} z^{\nu-1}, \quad|z|<1
$$

Because of (21) we obtain

$$
T\left(f^{*}(z)\right)=\sum_{\nu=1}^{\infty} \Omega_{\nu} z^{\nu}+\text { const }+\kappa \sum_{\nu=1}^{\infty} \bar{\Omega}_{\nu} z^{-\nu} \quad 1<|z|<r .
$$

By choosing $\theta=0$ it is easy to see that equality in (19) holds for $f^{*}(z)$. Because of (20) the inequality $\lambda_{2} \geq 1 / \kappa$ follows from a known result of L.V. Ahlfors (see [1]); consequently $f^{*}(z) \in \sum\langle\kappa\rangle$.

Suppose now that equality in (19) holds for any mapping $f(z) \in \sum\langle\kappa\rangle$. Then the function $H(w)$ in (7) must be a real eigenfunction belonging to $1 / \kappa$. This means that $e^{-i \theta} \kappa T(w)-e^{i \theta} \overline{T(w)}$ can be extended to an analytic function in the complement of $f(\{z:|z|>1\})$ (see [12; Theorem 5]). Consequently, it follows from (18) that

$$
\omega_{\nu}=\kappa e^{2 i \theta} \bar{\Omega}_{\nu}, \quad \nu=1,2, \ldots
$$

It can be proved as above that a suitable continuation of $f(z)$ satisfies

$$
f_{\bar{z}}(z)=\kappa e^{2 i \theta} \frac{\overline{T^{\prime}(z)}}{\overline{T^{\prime}(z)}} f_{z}(z)
$$

Summarizing these facts, we can state our next result. 
Theorem 2. If the analytic function $T(w), w \in(f(\{z:|z|>1\}))^{C}$, satisfies

$$
T(f(z))=\sum_{\nu=1}^{\infty} \Omega_{\nu} z^{\nu}+\text { const }+\sum_{\nu=1}^{\infty} \omega_{\nu} z^{-\nu}
$$

for all mappings $f(z) \in \sum\langle\kappa\rangle$, where the numbers $\Omega_{\nu}$ are fixed, then equality in

$$
\sum_{\nu=1}^{\infty} \nu\left|\omega_{\nu}+\frac{1}{2}(1-\kappa) e^{2 i \theta} \bar{\Omega}_{\nu}\right|^{2} \leq\left(\frac{1}{2}(1+\kappa)\right)^{2} \sum_{\nu=1}^{\infty} \nu\left|\Omega_{\nu}\right|^{2}
$$

holds only for the functions $f^{*}(z) \in \sum\langle\kappa\rangle$ which can be extended to solutions of

$$
f_{\bar{z}}^{*}(z)=\kappa e^{2 i \theta} \frac{\overline{T^{\prime}(z)}}{T^{\prime}(z)} f_{z}^{*}(z), \quad|z|<1 .
$$

\section{Considerations of multiply connected domains}

It will be shown that the inequality (11) is, in general, unsharp for multiply connected domains. For the sake of simplicity, we investigate only the case $T(w)=$ $w$. Suppose that equality (11) holds for any mapping $f^{*}(z) \in \sum\langle\kappa\rangle$. As before, we conclude from (8) that $\kappa e^{-i \theta} f^{*}(z)-e^{i \theta} \overline{f^{*}(z)}+$ const can be extended to an analytic function in $G$. This provides

$$
f^{*}(z)=a_{11} \Phi_{1}(z)+\kappa a_{11} e^{2 i \theta} \varphi_{1}(z)+\text { const }, \quad z \in G,
$$

where $a_{11}$ is a positive constant depending only on the domain $G$ (see [16; Chapter 5, Section 2]).

Considering the example of an annulus (see Section 3), we get by using the harmonic function with the boundary values $1 /(\bar{z}-1)$

$$
f^{*}(z)-\frac{1}{z-1}=\sum_{\nu=1}^{\infty} \frac{R^{4 \nu}}{1-R^{4 \nu}}\left(z^{-\nu}-z^{\nu}\right)+\kappa e^{2 i \theta} \sum_{\nu=1}^{\infty} \frac{R^{2 \nu}}{1-R^{4 \nu}}\left(z^{\nu}-z^{-\nu}\right)+\text { const. }
$$

It is easy to see that equality in (17) holds for $f^{*}(z)$. But the question whether $f^{*}(z)$ belongs to the class $\sum\langle G, \kappa\rangle$ remains still open. To find an answer for this question we shall prove an extremal property of circular domains.

Theorem 3. If the doubly connected domain $G^{*}$ bounded by two Jordan curves is conformally equivalent to the annulus $\{w: R<|w|<1 / R\}, 0<R<1$, then the Fredholm eigenvalue $\lambda_{2}$ of $G$ fulfills

$$
\lambda_{2} \leq \frac{1}{R^{2}} .
$$

Equality can occur in (26) if, and only if, $G^{*}$ is a circular domain. 
Proof. Denoting by $g(z)$ a homeomorphic and conformal mapping of $G^{*}$ onto $\{w: R<|w|<1 / R\}$, we shall estimate the quotient of Dirichlet's integrals in (3) for the function

$$
h(z)= \begin{cases}g(z)+1 / \overline{g(z)}, & \text { if } z \in G^{*} \\ h_{1}(z) & \text { if } z \in B_{1} \\ h_{2}(z) & \text { if } z \in B_{2}\end{cases}
$$

where $B_{1}$ is the component of $G^{* C}$ bounded by the inverse image of $\{w:|w|=R\}$ and $B_{2}$ is the other component of $G^{* C}$. It follows from the definition of $g(z)$ that

$$
\iint_{G^{*}}\left(\left|h_{z}(z)\right|^{2}+\left|h_{\bar{z}}(z)\right|^{2}\right) d x d y=2 \pi\left(\frac{1}{R^{2}}-R^{2}\right) .
$$

Let $h_{1}(z)$ be the harmonic function in $B_{1} \backslash \partial B_{1}$ which satisfies

$$
h_{1}(z)=g(z)+1 / \overline{g(z)}=g(z) \cdot\left(1+1 / R^{2}\right)
$$

on the boundary of $B_{1}$. This implies that

$$
\begin{aligned}
\iint_{B_{1}} & \left(\left|h_{1 z}(z)\right|^{2}+\left|h_{1 \bar{z}}(z)\right|^{2}\right) d x d y \\
& \geq \iint_{B_{1}}\left(\left|h_{1 z}(z)\right|^{2}-\left|h_{1 \bar{z}}(z)\right|^{2}\right) d x d y \\
& =\pi\left(1+\frac{1}{R^{2}}\right)^{2} R^{2} .
\end{aligned}
$$

If $h_{2}(z)$ is the harmonic function in the interior of $B_{2}$ which fulfills

$$
h_{2}(z)=g(z)+\frac{1}{\overline{g(z)}}=\left(1+\frac{1}{R^{2}}\right) \cdot \frac{1}{\overline{g(z)}}
$$

on the boundary of $B_{2}$, then

$$
\begin{aligned}
\iint_{B_{2}} & \left(\left|h_{2 z}(z)\right|^{2}+\left|h_{2 \bar{z}}(z)\right|^{2}\right) d x d y \\
& \geq \iint_{B_{2}}\left(\left|h_{2 \bar{z}}(z)\right|^{2}-\left|h_{2 z}(z)\right|^{2}\right) d x d y \\
& =\pi\left(1+\frac{1}{R^{2}}\right)^{2} R^{2} .
\end{aligned}
$$


Because of (28), (29) and (30) an application of the second inequality in (3) leads to

$$
\frac{2 \pi\left(1-R^{4}\right) / R^{2}}{\pi R^{2}\left(1+1 / R^{2}\right)^{2}+\pi R^{2}\left(1+1 / R^{2}\right)^{2}} \geq \frac{\lambda_{2}-1}{\lambda_{2}+1} .
$$

This proves (26). Equality in (26) can occur if, and only if, $h_{1 \bar{z}}(z)=0$ and $h_{2 z}(z)=0$ holds in $B_{1}$ and $B_{2}$, respectively. Consequently, $h_{1}(z)$ and $\overline{h_{2}(z)}$ are analytic functions. Since $h_{2}(z)=\left(1+1 / R^{2}\right) / \overline{g(z)}$ on the boundary of $B_{2}$, it follows from the argument principle that $h_{2}(z)$ has only one simple zero in $B_{2}$. Hence $g(z)$ is a linear fractional transformation. This completes the proof.

Now we consider again our example of an annulus. By choosing $\kappa=R^{2}$ we conclude from Theorem 3 that $\sum\langle G, \kappa\rangle$ consists only of the mappings

$$
f(z)=\frac{1}{z-1}+\text { const. }
$$

On the other hand, equality in (17) can occur only for the mappings $f^{*}(z)$ in (25). This contradiction shows that (17) provides an unsharp estimate for all $\kappa$ $\left(1>\kappa \geq R^{2}\right.$ ) sufficiently close to $R^{2}$. Furthermore, the condition $\kappa \geq R^{2}$ is also necessary for $\sum\langle G, \kappa\rangle \neq \emptyset$ when $G$ is a doubly connected domain having the conformal modulus $1 / R^{2}$.

\section{Remarks}

1. From Theorem 1 some estimations of functionals defined on $\sum\langle G, \kappa\rangle$ may be derived. Suppose that the numbers $\Gamma_{\nu}, \nu=1,2, \ldots$, in (5) do not depend on the mapping $f(z) \in \sum\langle G, \kappa\rangle$. Then the expression

$$
\sum_{\nu=1}^{\infty} \Gamma_{\nu} \gamma_{\nu}
$$

defines a functional for all mappings $f(z) \in \sum\langle G, \kappa\rangle$. Now Theorem 1 leads to

$$
\left|\sum_{\nu=1}^{\infty} \Gamma_{\nu} \gamma_{\nu}\right| \leq \kappa \sum_{\nu=1}^{\infty}\left|\Gamma_{\nu}\right|^{2} ;
$$

in fact, Schwarz's inequality yields

$$
\begin{aligned}
\sum_{\nu=1}^{\infty} \mid \gamma_{\nu} & +\left.\frac{1}{2}(1-\kappa) e^{2 i \theta} \bar{\Gamma}_{\nu}\right|^{2} \sum_{\nu=1}^{\infty}\left|\bar{\Gamma}_{\nu}\right|^{2} \\
& \geq\left.\left.\left|\sum_{\nu=1}^{\infty} \gamma_{\nu} \Gamma_{\nu}+\frac{1}{2}(1-\kappa) e^{2 i \theta} \sum_{\nu=1}^{\infty}\right| \Gamma_{\nu}\right|^{2}\right|^{2} \\
& \geq\left(\operatorname{Re}\left(e^{-2 i \theta} \sum_{\nu=1}^{\infty} \gamma_{\nu} \Gamma_{\nu}+\frac{1}{2}(1-\kappa) \sum_{\nu=1}^{\infty}\left|\Gamma_{\nu}\right|^{2}\right)\right)^{2}
\end{aligned}
$$


and thus we get from (11)

$$
\operatorname{Re}\left(e^{-2 i \theta} \sum_{\nu=1}^{\infty} \gamma_{\nu} \Gamma_{\nu}\right) \leq \kappa \sum_{\nu=1}^{\infty}\left|\Gamma_{\nu}\right|^{2}
$$

In general, (32) is an unsharp inequality. For simply connected domains $G$, however, this estimate becomes a sharp one. The inequality (32) generalizes the inequalities (4), (6) and (7) in [13] which were proved in a similar way. It should be noted that the representation (31) is valid for some known functionals, for instance for the coefficient $\alpha_{1}$ in (1), for the Schwarzian derivative and for Golusin's functional (see [6], [7] and [8]). The estimate of the range of $\alpha_{1}$ has the form

$$
\left|\alpha_{1}-m\right| \leq \kappa a_{11}^{2}
$$

where $m, a_{11}^{2}$ are the centre and the radius of the circular disk which belongs to the class $\sum(G)$. Note that the extremal mapping $f^{*}(z)$ in Theorem 2 also fulfills

$$
\left(T\left[f^{*}(z)\right]\right)_{\bar{z}}=\kappa e^{2 i \theta} \overline{\left(T\left[f^{*}(z)\right]\right)_{z}}, \quad|z|<1 .
$$

2. In Section 3 we have considered the example of an annulus. We point out that the series on the right-hand side of (17) satisfies

$$
\sum_{\nu=1}^{\infty} \frac{\nu R^{2 \nu}}{1-R^{4 \nu}}=\frac{1}{2 \pi^{2}} \mathbf{K}(k)(\mathbf{K}(k)-\mathbf{E}(k)),
$$

where $\mathbf{E}(k)$ and $\mathbf{K}(k)$ are the known complete elliptic integrals (see [18; p. 693, 5.1.29.3]) and $k$ fulfills

$$
R^{2}=\exp \left[-\pi \frac{\mathbf{K}\left(\sqrt{1-k^{2}}\right)}{\mathbf{K}(k)}\right]
$$

The last term is closely related to the conformal modulus of Grötzsch's domain (see [5] and [15, Chapter II, Sections 1 and 2]).

3. In this paper the derived inequalities do not contain coefficients of some unknown (extremal) mappings unlike the area theorems in [6] and [7]. On the other hand, the area theorems in [6] and [7] are also sharp for multiply connected domains $G$. This illustrates the shortcoming of the area theorem in Section 2. A common property of these area theorems in [6], [7] and in this paper is, however, that the area theorem for mappings $f(z) \in \sum(G)$ (see [16; Theorem 5.1]) can be interpreted as a special case of them. 


\section{References}

[1] Ahlfors, L.V.: Remarks on the Neumann-Poincare integral equation. - Pacific J. Math. 2, 1952, 271-280.

[2] Ahlfors, L.V.: A remark on schlicht functions with quasiconformal extensions. - Symposium on Complex Analysis, Canterbury, 1973. London Mathematical Society Lecture Note Series 12; edited by J. Clunie and W.K. Hayman. Cambridge University Press, 1974, 7-10.

[3] Anderson, J.M.: The Fredholm eigenvalues of plane domains. - Complex Variables Theory Appl. 5, 1986, 111-116.

[4] Gaier, D.: Konstruktive Methoden der konformen Abbildung. - Springer-Verlag, BerlinGöttingen-Heidelberg, 1964.

[5] Grötzsch, H.: Über einige Extremalprobleme der konformen Abbildung. - Ber. Math.phys. Kl. Sächs. Akad. Wiss. Leipzig 80, 1928, 367-376.

[6] Hoy, E.: Flächensätze für quasikonform fortsetzbare Abbildungen. - Z. Anal. Anwendungen 3 (1), 1984, 19-31.

[7] Hoy, E.: Flächensätze für quasikonform fortsetzbare Abbildungen mit ortsabhängiger Dilatationsbeschränkung. - Math. Nachr. 121, 1985, 147-161.

[8] HoY, E.: Zur Abschätzung von einigen Funktionalen mit Hilfe der Randintegration. - Wiss. Z. Martin-Luther-Univ. Halle-Wittenberg, Math.-Nat. Reihe 35 (4), 1986, 155-161.

[9] Kruschkal, S.L., and R. KühnaU: Quasikonforme Abbildungen - neue Methoden und Anwendungen. - Teubner-Verlag, Leipzig, 1985. In Russian: "Nauka", Novosibirsk, 1984.

[10] Krzyż, J.G.: Generalized Fredholm eigenvalues of a Jordan curve. - Ann. Polon. Math. $46,1985,157-163$.

[11] KühnaU, R.: Verzerrungssätze und Koeffizientenbedingungen vom Grunskyschen Typ für quasikonforme Abbildungen. - Math. Nachr. 48, 1971, 77-125.

[12] KüHNAU, R.: Eine Integralgleichung in der Theorie quasikonformen Abbildungen. - Math. Nachr. 76, 1977, 139-152.

[13] Kühnau, R.: Zu den Grunskyschen Coeffizientenbedingungen. - Ann. Acad. Sci. Fenn. Ser. A I Math. 6, 1981, 125-130.

[14] Lento, O.: Schlicht functions with a quasiconformal extension. - Ann. Acad. Sci. Fenn. Ser. A I Math. 500, 1971, 1-10.

[15] Lehto, O., and K.I. Virtanen: Quasikonforme Abbildungen. - Springer-Verlag, BerlinHeidelberg-New York, 1965.

[16] MiLin, I.M.: Univalent functions and orthonormal systems. - American Mathematical Society, Providence, R.I., 1977.

[17] Pommerenke, Ch.: Univalent functions. - Vandenhoeck \& Ruprecht, Göttingen, 1975.

[18] Prudnikov, A.P., Yu.A. Brychkov, and O.I. Marichev: Integrals and series. Elementary functions. - "Nauka", Moscow, 1981 (Russian).

[19] SCHIfFer, M.: Fredholm eigenvalues of multiply-connected domains. - Pacific J. Math. 9, 1959, 211-269. 
[20] Schiffer, M., and G. SPRINGer: Fredholm eigenvalues and conformal mapping of multiply connected domains. - J. Analyse Math. 14, 1965, 337-378.

[21] Springer, G.: Fredholm eigenvalues and quasiconformal mapping. - Acta Math. 111, 1964, 121-142.

Martin-Luther-Universität Halle-Wittenberg

Sektion Mathematik

4010 Halle

German Democratic Republic

Received 21 March 1988 